\title{
RhoGEF Trio Regulates Radial Migration of Projection Neurons via Its Distinct Domains
}

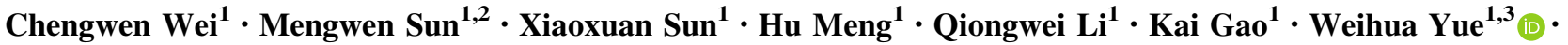 \\ Lifang Wang ${ }^{1} \cdot$ Dai Zhang ${ }^{1,4,5}\left(\mathbb{D} \cdot\right.$ Jun $^{\mathrm{Li}^{1}}$
}

Received: 23 February 2021 / Accepted: 28 September 2021 / Published online: 16 December 2021

(C) The Author(s) 2021

\begin{abstract}
The radial migration of cortical pyramidal neurons (PNs) during corticogenesis is necessary for establishing a multilayered cerebral cortex. Neuronal migration defects are considered a critical etiology of neurodevelopmental disorders, including autism spectrum disorders (ASDs), schizophrenia, epilepsy, and intellectual disability (ID). TRIO is a high-risk candidate gene for ASDs and ID. However, its role in embryonic radial migration and the etiology of ASDs and ID are not fully understood. In this study, we found that the in vivo conditional knockout or in utero knockout of Trio in excitatory precursors in the neocortex caused aberrant polarity and halted the migration of late-born PNs. Further
\end{abstract}

Supplementary Information The online version contains supplementary material available at https://doi.org/10.1007/s12264-02100804-7.

Dai Zhang

daizhang@bjmu.edu.cn

$\triangle$ Jun Li

junli1985@bjmu.edu.cn

1 Peking University Sixth Hospital, Peking University Institute of Mental Health, NHC Key Laboratory of Mental Health (Peking University), National Clinical Research Center for Mental Disorders (Peking University Sixth Hospital), Beijing 100191, China

2 Peking-Tsinghua Center for Life Sciences, Peking University, Beijing 100871, China

3 PKU-IDG/McGovern Institute for Brain Research, Peking University, Beijing 100871, China

4 Chinese Institute for Brain Research, Beijing 100010, China

5 Institute for Brain Research and Rehabilitation (IBRR), Guangdong Key Laboratory of Mental Health and Cognitive Science, South China Normal University, Guangzhou 510631, China investigation of the underlying mechanism revealed that the interaction of the Trio N-terminal SH3 domain with Myosin X mediated the adherence of migrating neurons to radial glial fibers through regulating the membrane location of neuronal cadherin (N-cadherin). Also, independent or synergistic overexpression of RAC1 and RHOA showed different phenotypic recoveries of the abnormal neuronal migration by affecting the morphological transition and/or the glial fiber-dependent locomotion. Taken together, our findings clarify a novel mechanism of Trio in regulating $\mathrm{N}$-cadherin cell surface expression via the interaction of Myosin X with its N-terminal SH3 domain. These results suggest the vital roles of the guanine nucleotide exchange factor 1 (GEF1) and GEF2 domains in regulating radial migration by activating their Rho GTPase effectors in both distinct and cooperative manners, which might be associated with the abnormal phenotypes in neurodevelopmental disorders.

Keywords Trio - Myosin X - RhoGEF - Neurodevelopmental disorder $\cdot$ Radial migration

\section{Introduction}

During neocortical development, the highly organized layering is achieved via the radial migration of neurons from the ventricular zone (VZ) to the destined layers in an inside-out manner [1]. The precursors of pyramidal neurons (PNs) undergo a multipolar (MP) to bipolar (BP) morphological transition as they migrate from the lower to the upper intermediate zone (IZ). BP PNs migrate radially into the cortical plate (CP) through glial-guided locomotion, then undergo somal translocation to reach their final position, where they are wired into functional circuits 
$[2,3]$. Neuronal migration deficits are considered to be an important etiology of many neurodevelopmental disorders, including autism spectrum disorders (ASDs), schizophrenia, epilepsy, and intellectual disability (ID) [4-8]. However, the mechanisms underlying neuronal migration deficits in the etiology of these diseases remain unclear.

TRIO (trio Rho guanine nucleotide exchange factor) encodes a guanine nucleotide exchange factor (GEF) that facilitates the activation of Rho GTPases. Previous studies mainly focused on the roles of Trio in postmitotic PNs, including axon guidance [9, 10], dendritic branching, and synaptic transmission and plasticity [11, 12]. Although the olfactory bulb, hippocampus, and cerebellum develop abnormally upon Trio deletion [13, 14], the role of Trio in the embryonic development of the neocortex, especially in the radial migration of $\mathrm{PNs}$, is unclear. The contribution of Rho GEFs to the pathogenesis of neuropsychiatric disorders, including ASDs, was recently reported [15]. Moreover, a cluster of deleterious de novo mutations in TRIO GEF1 and the spectrin repeat domain were recently identified in neurodevelopmental disorders including ASDs and/or ID [16-18], suggesting that Trio dysfunction is a risk factor for neurodevelopmental diseases. However, little attention has been devoted to the involvement of Triomediated cortical radial migration in the etiology of ASDs and ID. Furthermore, Trio contains two independent GEF domains for the specific activation of Rac1/RhoG (GEF1/ N-terminal GEF domain) and RhoA (GEF2/C-terminal GEF domain) [19-21]. Both the DH1 and PH1 domains in GEF1 play a role in Rac1 interaction and activation as previously reported [22]. The N-terminal SH3 has been reported to modulate GEF1 activity but is not required for Rac1 or RhoG guanosine diphosphate (GDP)-guanosine triphosphate (GTP) exchange [23, 24]. However, the roles of different domains of Trio in embryonic radial migration are largely unknown and need further clarification.

In this study, the function of Trio in cortical migration was addressed in vivo through the deletion of Trio in excitatory progenitors using in utero electroporation of Cre recombinase. The morphogenesis and migratory behaviors of the Trio-ablated neurons were investigated by immunofluorescence against various markers. Trio promoted neuronal morphogenesis during the MP-to-BP transition in the IZ and the extension of the leading process of late-born neurons. In addition, the interaction of Trio N-terminal SH3 with Myosin X was essential for radial glial-dependent migration through regulating the neuron-glia adhesion mediated by the membrane location of $\mathrm{N}$-cadherin in the $\mathrm{CP}$. Trio promoted the radial migration via its GEF1 and GEF2 domains in the different Rho GTPase signaling pathways. Our study on the function of Trio in embryonic radial migration provides new insights into the involvement of neuronal migration in the pathogenesis of neurodevelopment disorders.

\section{Materials and Methods}

\section{Animals}

Trio $^{\text {flfl }}$ mice were purchased from the Model Animal Research Center (Nanjing, China) as previously described [14] and were backcrossed with C57BL/6 mice for $>10$ generations. For in utero electroporation, the Trio ${ }^{f l / f l} \mathrm{C} 57 /$ BL6 mice were crossed with ICR mice to obtain a Trio ${ }^{f / f t}$ inbred ICR strain. Emx1-Cre mice were crossed with Trio $^{f / f l}$ mice to generate Trio ${ }^{f / f l: E m x-C r e}$ mice. All animal experiments were performed in strict accordance with the Peking University institutional animal care and use guidelines and approved by the Animal Care Committee.

\section{Antibodies}

The primary antibodies used were as follows: rabbit antiTrio (Santa Cruz, sc-28564, 1:50), rabbit anti-GFP (Invitrogen, A1112, 1:2000), mouse anti-GFP (Invitrogen, MA515256, 1:200), mouse anti-Nestin (Millipore, MAB353, 1:100), rabbit anti-GRASP65 (Abcam, ab174834, 1:1000), rabbit anti-N-cadherin (Cell Signaling Technology, 3116s, 1:100), rabbit anti-Ki67 (Abcam, AB15580, 1:500), mouse anti-Tuj1 (Sigma-Aldrich, T8660, 1:1000), rabbit antiCux1 (Santa Cruz, sc-13024, 1:200), rabbit anti-Tbr1 (Abcam, ab31940, 1:1000), rabbit anti-caspase-3 (Cell Signaling Technology, P42574, 1:500), mouse anti-Flag (Sigma-Aldrich, F3165, 1:2000), rabbit anti-HA (SigmaAldrich, 05-902R, 1:2000), and rabbit anti-GAPDH (Cell Signaling Technology, 5174, 1:2000). F-actin was stained with Alexa-555 phalloidin (Invitrogen, A34055, 1:100). The secondary antibodies used were as follows: Alexa 488 anti-rabbit (Invitrogen, A11008, 1:1000), Alexa 555 antirabbit (Invitrogen, A21428, 1:1000), Alexa 488 anti-mouse (Invitrogen, A11001, 1:1000), and Alexa 555 anti-mouse (Invitrogen, A21422, 1:1000).

\section{Plasmid Construction}

The following constructs were used for in utero electroporation and transfection in primary cultured neurons: pcAGGS-Cre-HA-IRES-EGFP, pNeuroD1-Cre-IRES-EGFP, pcAGGS-MYOSIN X-3Flag-IRES-EGFP, pcDNA3.1-TRIO 9S-HA, pcDNA3.1-TRIO9S-ASP-HA, pcDNA3.1-TRIO9S$\triangle$ GEF1-SH3-HA, pcDNA3.1-TRIO9S- $A G E F 1-H A, \quad p c D N$ A3.1-TRIO9S- $A G E F 2-H A, \quad p c A G G S-R H O A-I R E S-E G F P$, pcAGGS-RAC1-IRES-EGFP, pcAGGS-CA/constitutively active (CA)]-PAK1-HA-IRES-EGFP, and pcAGGS-CA- 
ROCK-HA-IRES-EGFP. The human Myc-TRIO-FL plasmid was a gift from Prof. Richard E. Mains (University of Connecticut Health Center, MI, USA). The TRIO9S-HA plasmid was constructed based on the Myc-Trio-FL plasmid. The TRIO9S- $\Delta$ GEF1-SH3-HA, TRIO9S- $\Delta$ GEF1-HA, TRIO 9S- $\Delta$ GEF2-HA, and TRIO9S- $\Delta$ SP-HA plasmids were further constructed to delete the GEF1-SH3, GEF1, GEF2, and SP domains based on the TRIO9S-HA plasmid, respectively. Human MYOSIN X, RAC1, RHOA, and CA mutants of PAK1 and ROCK were amplified from cDNA and cloned into the $p c A G G S-I R E S-E G F P$ plasmid.

\section{Co-immunoprecipitation and Immunoblotting}

HEK293T cells were cultured in Dulbecco's modified Eagle's medium (DMEM) supplemented with $10 \%$ fetal bovine serum (FBS), $100 \mathrm{U} / \mathrm{mL}$ penicillin, and $100 \mathrm{mg} / \mathrm{mL}$ streptomycin, transfected with appropriate expression plasmids using jetOPTIMUS (Polyplus-transfection, France), and incubated in a humidified $5 \% \mathrm{CO}_{2}$ atmosphere at $37^{\circ} \mathrm{C}$ for $48 \mathrm{~h}$. The cells were harvested in phosphate-buffered saline (PBS), spun down, and re-suspended on ice with lysis buffer $[50 \mathrm{mmol} / \mathrm{L}$ Tris/ $\mathrm{HCl}(\mathrm{pH} 8.0), 200 \mathrm{mmol} / \mathrm{L}$ $\mathrm{NaCl}, 1 \%$ Triton $\mathrm{X}-100$, complete proteinase inhibitors (Roche), and EDTA]. The proteins were solubilized by rotating the cell lysates at $4^{\circ} \mathrm{C}$ for $20 \mathrm{~min}$ on a rotating wheel, and the supernatants were then cleared by centrifugation at $12,000 \mathrm{~g}$ for $15 \mathrm{~min}$ at $4^{\circ} \mathrm{C}$. The supernatant lysate was incubated with $2 \mu \mathrm{g}$ anti-HA or anti-FLAG co-injected beads overnight at $4^{\circ} \mathrm{C}$. The beads were washed four times with the lysis buffer. The bound proteins were eluted with $2 \times$ Laemmli sample buffer (Sigma-Aldrich) and loaded onto sodium dodecyl sulfate-polyacrylamide gel electrophoresis (SDS-PAGE) for immunoblotting using rabbit anti-HA (1:2000) and mouse anti-Flag (1:2000). For brain tissue, the E14.5 cortex was homogenized with RIPA [50 $\mathrm{mmol} / \mathrm{L}$ Tris $(\mathrm{pH} 7.4), 150 \mathrm{mmol} / \mathrm{L} \mathrm{NaCl}, 1 \%$ Triton $\mathrm{X}-100$, and $0.1 \%$ SDS] supplemented with protease inhibitors (Roche). The lysates were centrifuged at $12,000 \mathrm{~g}$ for $20 \mathrm{~min}$ at $4^{\circ} \mathrm{C}$. The supernatants were collected and boiled with $2 \times$ Laemmli sample buffer (Sigma-Aldrich) and loaded onto SDS-PAGE. After membrane transfer, rabbit anti-Trio (1:500), mouse antiGAPDH (1:2000), and HRP-conjugated secondary antibodies (1:1000) were used for immunoblotting. The membranes were developed and imaged using the enhanced chemiluminescence reagent (Thermo Fisher Scientific). To quantify the intensity of bands of interest, three independent experiments for each molecule were analyzed using ImageJ software (NIH). GAPDH was used as an internal control to normalize band intensity.

\section{Immunofluorescence}

Pregnant mice were anesthetized, and the embryos were perfused with $4 \%(w / v)$ paraformaldehyde (SigmaAldrich) in PBS. The brains were removed and post-fixed overnight at $4{ }^{\circ} \mathrm{C}$, following dehydration in $25 \%(\mathrm{w} /$ v) sucrose diluted in PBS for 2 days at $4^{\circ} \mathrm{C}$. The brains were embedded an optimum cutting temperature compound and sectioned coronally at $25 \mu \mathrm{m}$ on a cryostat (Leica Biosystems, Germany). The sections were treated with citrate antigen retrieval solution (Beyotime, China) at $80^{\circ} \mathrm{C}$ for $12 \mathrm{~min}$ if necessary. After three times washing with $1 \times$ PBS for 10 min each time, the sections were blocked with $5 \%(w / v)$ bovine serum albumin and $0.1 \%$ Triton X-100 in PBS for 30 min at room temperature and incubated at $4^{\circ} \mathrm{C}$ with the indicated primary antibodies for $18 \mathrm{~h}$. The sections were then rinsed three times with $0.01 \%$ Triton X-100 in PBS for 10 min each and incubated with Alexa Flour 488 and 555 for $1 \mathrm{~h}$ at room temperature. The nuclei were stained using Hoechst 33342 (Invitrogen) at room temperature for $10 \mathrm{~min}$. The sections were imaged with an FV-1200 confocal laser scanning biological microscope (Olympus, Japan) and an IX71 inverted fluorescence microscope (Olympus, Japan).

\section{In Utero Electroporation}

Pregnant mice were deeply anesthetized, and the intrauterine embryos were surgically manipulated as described previously [25]. In brief, $p c A G G S$ carrying $C r e$ and internal ribozyme entry site (IRES) driving enhanced green fluorescent protein $(E G F P)$ was purified without endotoxin. The concentration of the plasmid was adjusted to $2 \mathrm{mg} / \mathrm{mL}$. The plasmid containing $0.02 \%$ Fast Green solution was injected into the lateral ventricle of embryos at the indicated time. The plasmids were delivered into the VZ surface of the $\mathrm{CP}$ in the somatosensory cortical region by electronic pulses (Nepa Gene, Japan). The operated embryos were allowed to live within the uterine horn until the time of observation.

\section{Brain Slice Culture}

For embryonic brain slice culture, the brains were dissected out in cold Hibernate-E medium (Gibco). Brain slices (300 $\mu \mathrm{m}$ thick) from E15.5 embryos electroporated with the indicated plasmids at E13.5 were prepared with a Leica Vibratome VT1200. To visualize neuronal migration, the slices were transferred onto Millicell inserts (Millipore) in Neurobasal medium (Gibco) containing 20\% FBS, $2 \mathrm{mmol} / \mathrm{L}$ L-glutamine, and penicillin/streptomycin (50 U/50 $\mu \mathrm{g} / \mathrm{mL}$ ). The glass-bottomed dish was then fitted into a temperature-controlled chamber on the microscope 
stage and incubated for $16 \mathrm{~h}$ at $37^{\circ} \mathrm{C}$ under a $5 \% \mathrm{CO}_{2}$ air atmosphere. Live cell images were captured using an Olympus FV1200 Viewer laser scanning confocal microscope.

\section{Primary Neuronal Culture}

The cortical tissue from E16.5 embryos electroporated with the indicated plasmids at E14.5 were digested with $0.125 \%$ trypsin at $37^{\circ} \mathrm{C}$ for $15 \mathrm{~min}$ and dissociated by pipetting in DMEM with $20 \%$ FBS. The medium was changed to Neurobasal medium (Gibco) with 2\% B27 supplement (Gibco) and $2 \mathrm{mmol} / \mathrm{L}$ L-glutamine (Gibco) $2 \mathrm{~h}$ after seeding.

\section{Results}

\section{Trio Regulates Radial Migration and Leading Pro- cess Morphology of Late-born Neurons in the Em- bryonic Cortex}

We first examined the expression of Trio in the developing cortex by immunostaining to explore its role in cortical morphogenesis. Trio was co-localized with Tuj1, a $\beta$ tubulin marker specific to postmitotic neurons in the embryonic cortex (Fig. 1A), indicating that Trio might play a critical role in the cortical development. We then investigated Trio function in projection neurons during neocortical development. Progenitors in the VZ of Trio ${ }^{\text {fl/ }}$ embryos were electroporated with the chicken beta-actin (CAG) promoter-driven Cre-IRES-EGFP plasmid to delete Trio in early-born (E12.5) and late-born (E14.5) neurons. The CAG promoter-driven IRES-EGFP plasmid was used as a control. In Trio-deleted embryos, EGFP-positive $\left(\mathrm{EGFP}^{+}\right)$late-born cortical neurons abnormally accumulated in the IZ and fewer neurons were found in the upper $\mathrm{CP}$ at P0 (Fig. 1B). Trio ablation had no effect on the radial migration of early-born projection neurons (Fig. 1C). To exclude the possibility that the impaired radial migration was due to the effect of Trio deletion on progenitor proliferation and/or cell-cycle exit, the pNeuroD1-CreIRES-EGFP vector was designed and generated to allow Cre expression in early postmitotic neurons under the control of the mouse NeuroD1 promoter [26]. The E14.5 electroporated Trio ${ }^{\text {flfl:NeuroD1-Cre-IRES-EGFP }}$ neurons showed defective migration relative to the Trio fl/f:NeuroD1-IRES-EGFP neurons at E18.5 (Fig. 1D). Trio flfl:Emxl-Cre mice were generated by crossing Trio ${ }^{f / f l}$ mice with the Emxl-Cre line to delete Trio in the forebrain excitatory progenitors so as to further confirm the abnormal migration of neurons in the neocortex. Trio protein levels decreased significantly in the embryonic cortex of Trioflfl:Emx1-Cre mice compared with
Fig. 1 Depletion of Trio impairs embryonic radial migration of projection neurons. A High expression of Trio in postmitotic neurons. Tuj1 and Trio are co-labeled in the developing neurons of the ventricular and subventricular zones at E14.5. The dashed boxes in the upper panels are magnified in the lower panels. Nuclei stained with Hoechst. Scale bars, $50 \mu \mathrm{m}$ (upper panels) and $5 \mu \mathrm{m}$ (lower panels). B, C Left panels, endogenous Trio is knocked out by in utero electroporation (IUE) at E14.5 (B) and E12.5 (C) with overexpression of the pcAGGS-Cre-IRES-EGFP plasmid in the progenitors of floxedTrio mice using the pcAGGS-IRES-EGFP plasmid as a control. Nuclei stained with Hoechst to mark distinct CP, IZ, and SVZ/VZ layers. Scale bars, $50 \mu \mathrm{m}$. Right panels, the distribution and frequency of cortical neurons at P0 (B) and E15.5 (C) after electroporation ( $n=12$ slices from 3 Trio $^{\text {flftil:Ctl }}$ mice and $n=12$ slices from 3 Trio $^{\text {flff:Cre }}$ mice). D Left panels, endogenous Trio in postmitotic neurons is knocked out by IUE at E14.5 with the pNeuroD1-CreIRES-EGFP plasmid in floxed-Trio mice using the pNeuroD1-IRESEGFP plasmid as a control. Distinct CP, IZ, and SVZ/VZ layers are marked. Scale bar, $50 \mu \mathrm{m}$. Right panel, the distribution and frequency of the cortical neurons at E18.5 $(n=12$ slices from 3 Trio flff:NeuroDIIRES-EGFP mice and $n=12$ slices from 3 Trio flff:NeuroDI-Cre-IRES-EGFP mice). E, F Reduced protein level of Trio in the Trifolft:Emxl-Cre cortical cortex. Western blots at E15.5 showing Trio protein expression in Trio $o^{f / f l: E m x l-C r e}$ and Trio ${ }^{f / f l}$ cortex using the Trio antibody; GAPDH was used as an internal reference $(\mathbf{E})$. Relative Trio protein levels in the two groups $(\mathbf{F})\left(n=5\right.$ Trio $^{f / f l}$ mice and $n=5$ Trio ${ }^{f / f l: E m x l-C r e}$ mice). G, $\mathbf{H}$ Left panels, cortical sections of P0 Trio ${ }^{f /}$ fl:Emxl-Cre and Trio ${ }^{f l f l}$ mice immunostained using Cux1 and Tbr1 antibodies. Scale bar, $50 \mu \mathrm{m}$. Right panels, changes in the distribution of $\mathrm{Cux}^{+}$and $\mathrm{Tbr} 1^{+}$cells across 10 equal bins in the two groups. I-L Higher magnification images showing the morphology of the neuronal leading process in Trio ${ }^{f l f l: C r e}$ and Trio ${ }^{f l f l: C t l}$ neurons (I). Scale bar, 10 $\mu \mathrm{m}$. Statistical analysis of the number of processes $(\mathbf{J})$, total process length $(\mathbf{K})$, and length of a primary process extending from the soma (L) $\left(n=23\right.$ Trio $^{f l f l: C t l}$ neurons and $n=29$ Trio ${ }^{\text {flffl:Cre }}$ neurons $)$. Histograms show the mean $\pm \mathrm{SEM} ; * P<0.05 ; * * P<0.01$; $* * * P<0.001$, Student's $t$ test.

controls (Fig. 1E, F). The neocortex was immunostained for Cut like homeobox 1 (Cux1) and T-box brain transcription factor 1 (Tbr1), markers of neurons from cortical layers II-IV and VI, respectively (Fig. 1G, H). Consistent with the abnormal migration phenotypes of neurons labeled using electroporation, Cux1-positive $\left(\mathrm{Cux}^{+}{ }^{+}\right)$cells accumulated in the IZ of Triof/fl:Emxl-Cre mice at P0, leading to a lower proportion of neurons in the $\mathrm{CP}$ relative to the amounts found in control (Fig. 1G). In addition, the distribution of Tbr1-positive ( $\left.\mathrm{Tbr} 1^{+}\right)$neurons was not significantly different between the two genotypes (Fig. $1 \mathrm{H}$ ). The total length of the leading process and the length of the primary process extending from the soma were significantly shorter in the electroporated Trio $^{\text {flfl: }}$ Cre neurons than in Trio ${ }^{f / f l: C t l}$ neurons, while the number of processes was similar (Fig. 1I-L). In addition, the Trioflfl:Cre and Trio ${ }^{f / f l: C t l}$ neurons electroporated at E14.5 were also analyzed at P4. Trio-deleted neurons exhibited sustained location defects, as many more migratory $\mathrm{EGFP}^{+}$cells were localized in layers V-VI compared 
A
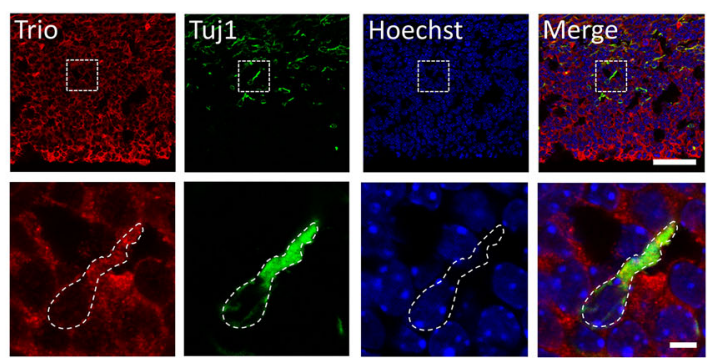

B
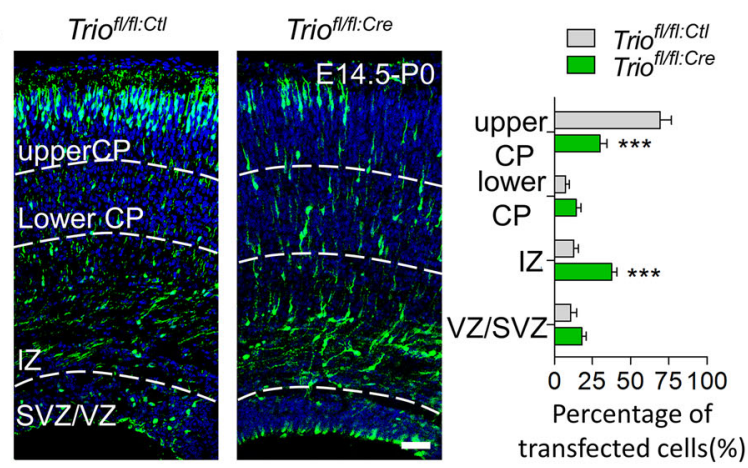

C
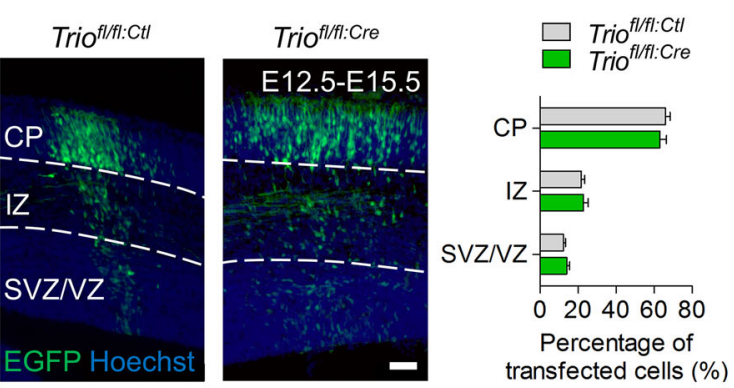

D

Trio fl/ffl:NeuroD1-IRES-EGFP Trio fl/fl:NeuroD1-Cre-IRES-EGFP
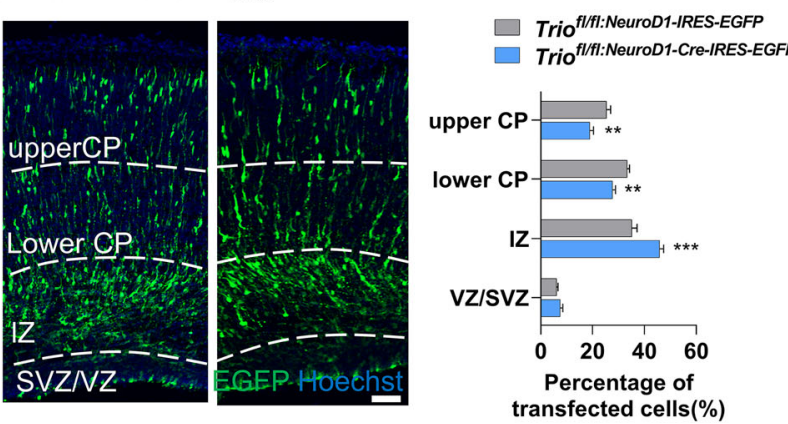

E

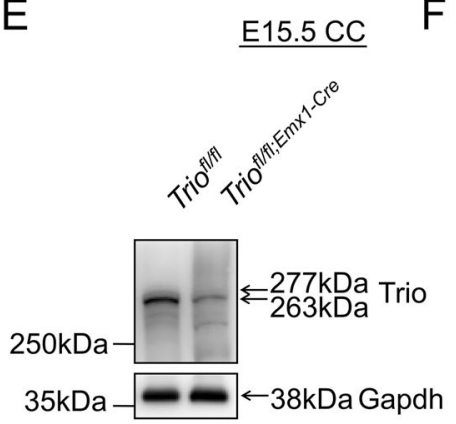

F
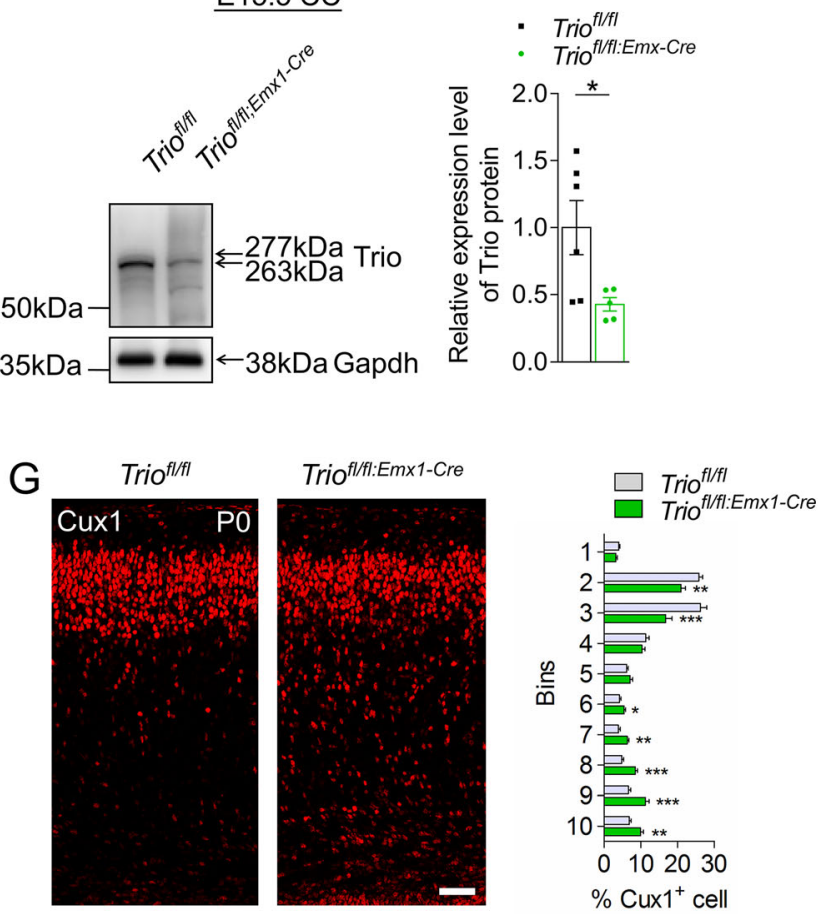

$\mathrm{H}$
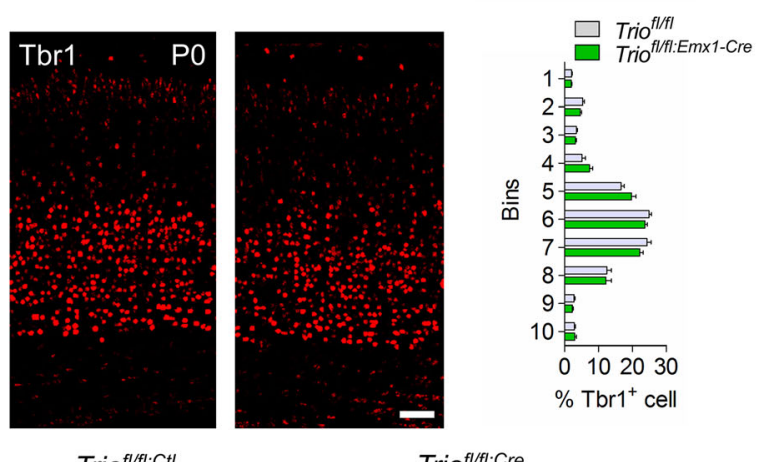

1 占

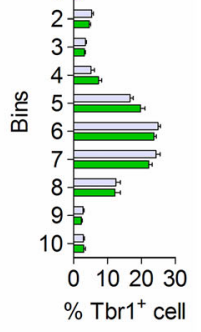

I

Trio

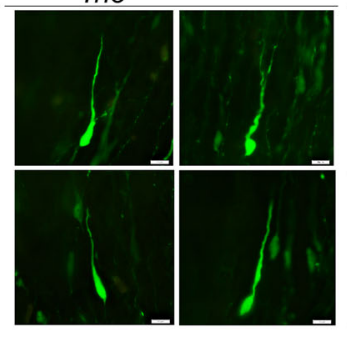

Trio $^{\text {fl/fl:Cre }}$

J
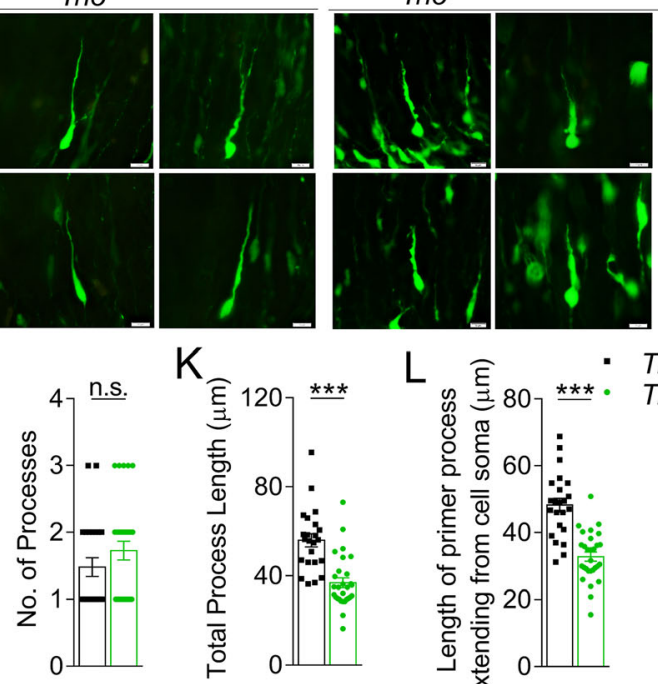

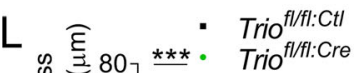

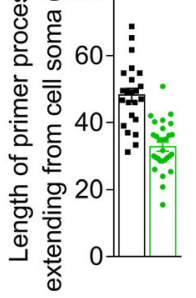


with the control neurons. This was consistent with the arrested neurons in the IZ during embryogenesis. A portion of the ectopic Trio ${ }^{f l f t: C r e}$ neurons was localized in the subcortical white matter (WM) (Fig. S1A). Morphological analysis showed abnormal leading processes and stunted neurites in Trio-depleted neurons in layers II-IV compared with controls (Fig. S1A). The extensions of axons from electroporated ipsilateral neurons to the contralateral side were shorter for Trio ${ }^{\text {flff:Cre }}$ neurons than for controls at P4 (Fig. S1B). Furthermore, the ectopic neurons present in the WM were positive for the layer II-IV marker Cux1, but negative for the layer VI marker Tbr1 (Fig. S1C). Altogether, these results suggested that Trio deletion impairs the morphogenesis and migration of late-born cortical projection neurons at embryonic and early postnatal development stages.

\section{Trio-deleted Projection Neurons Exhibit an Aber- rant Multipolar-to-Bipolar Transition and Migra- tion Velocity}

Both the morphological transition from MP-to-BP and the correct migratory orientation in the terminal location are important for the migration of late-born neurons. Trio ${ }^{f / f t: C t l}$ and Trio ${ }^{f / f l: C r e}$ neurons electroporated at E14.5 were analyzed at E16.5 to investigate neuronal morphology in the upper IZ. The proportion of unipolar/bipolar cells was lower in Trio ${ }^{f / f l: C r e}$ neurons than in controls (Fig. 2A), suggesting that the MP-to-BP transition is affected by Trio ablation. The Golgi apparatus marker GRASP65 was labeled to assess the migratory direction of neurons in the IZ. The proportion of Trio ${ }^{\text {flff:Cre }}$ neurons with the Golgi apparatus located toward the $\mathrm{CP}$ decreased in the IZ compared with Trio ${ }^{f l f l: C t l}$ neurons (Fig. 2B), which is in accordance with a defect in the MP-to-BP transition. In addition, time-lapse imaging of cortical slice cultures ex vivo was used to examine the migratory behaviors of cortical projection neurons. Trio ${ }^{f l f l: C t l}$ and Trio ${ }^{f l f t: C r e}$ slices, in which neuronal progenitors were electroporated at E13.5, were prepared at E15.5 and cultured for $8 \mathrm{~h}$ before a time-lapse imaging analysis. Images of the radial migration of projection neurons from the upper IZ to the $\mathrm{CP}$ were captured once every hour for a total of $6 \mathrm{~h}$ (Fig. 2C). Compared with Trio ${ }^{f / f t: C t l}$ neurons, most Trio $^{\text {flff:Cre }}$ neurons halted in the IZ and failed to move into the CP. In addition, Trio ${ }^{\text {flff:Cre }}$ neurons traveled shorter distances and exhibited a reduced migration speed (Fig. 2C). Immunostaining of the electroporated Trio $^{\text {flfl:Cre }}$ and Trio $^{\text {flfl:Ctl}}$ brains as early as 1 day after electroporation (E15.5) was used to assess cell-autonomous proliferation and apoptosis. The percentage of $\mathrm{EGFP}^{+}$cells co-stained with Ki67 cells among the total $\mathrm{EGFP}^{+}$cells did not differ between the two groups in the VZ, where the progenitors were actively dividing (Fig. S2A). In addition, no apoptotic $\mathrm{EGFP}^{+}$cells were present, as indicated by the absence of active caspase- 3 staining (Fig. S2B). These data suggested that Trio plays a role in the morphological transition of neurons and the migration velocity, but not in proliferation and apoptosis, in the early stages of the radial migration to the $\mathrm{CP}$.

\section{Trio Affects Neuronal Polarization by Regulating F-actin Polymerization}

The neurons from Trio-deleted and control cortex were extracted immediately after electroporation at E14.5 and cultured for 3 days to further explore the mechanisms induced by Trio deletion to regulate the MP-to-BP transition. During the neuronal polarization in vitro, nonpolar stage 1 neurons began to grow multiple short neurites to stage 2. A multipolar stage 2 neuron allowed one of its neurites to rapidly grow and become an axon-forming stage 3 neuron [27, 28]. The structural organization and dynamics of the growth cone are essential for neuronal polarization and migration. Thus, we evaluated the distribution of filamentous actin (F-actin) in the growth cones. The distribution of F-actin in the cellular extensions was limited in stage 1 Trio $^{\text {flff:Cre }}$ neurons compared with the control. The polymerized F-actin level decreased in the extended neurites of stage 2 Trio $^{\text {flff:Cre }}$ neurons. The decreased F-actin content resulted in shorter axon length at the polarized growth cone in the stage 3 Trio $^{\text {flft:Cre }}$ neuron (Fig. 3A). In addition, fewer stage 3 neurons and increased proportions of stage 1 and stage 2 neurons were observed in the Trio ${ }^{f l f l: C r e}$ group compared with the control (Fig. 3B). The average area of lamellipodia protrusion in stage 1 neurons was significantly smaller in Trio ${ }^{\text {flfl:Cre }}$ neurons than in control (Fig. 3C). Stage 2 Trio $^{\text {flfl:Cre }}$ neurons also had fewer neurites compared with controls (Fig. 3D). Decreased axon length (Fig. 3E) but a similar number of other neurites (Fig. 3F) were found in stage 3 Trio $^{\text {flfl:Cre }}$ neurons compared with controls. These results indicated that Trio ablation leads to decreased F-actin polymerization in the growth cone and then abnormal neuronal polarization.

\section{Trio Regulates Myosin X-dependent Cell Surface Localization of $\mathrm{N}$-cadherin to Promote Neural Adhesion to Radial Glial Fibers}

Alternative splicing of Trio gives rise to numerous isoforms [29]. The two isoforms without a C-terminal kinase domain, TRIO9S and TRIO9L, are considered the dominant isoforms expressed in the brain [30-32]. We investigated Trio interaction partners using mass spectrometry to explore other possible mechanisms underlying 
A
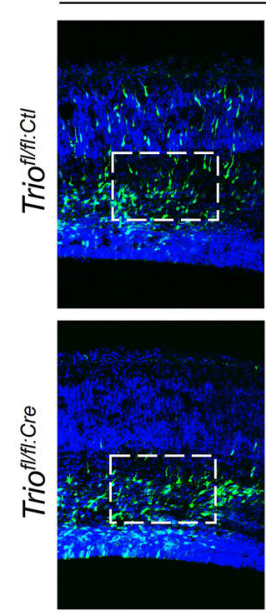

$\mathrm{E} 14.5-\mathrm{E} 16.5$
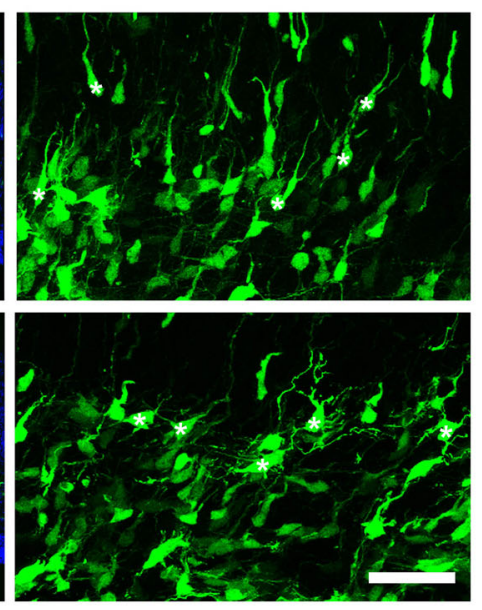

C

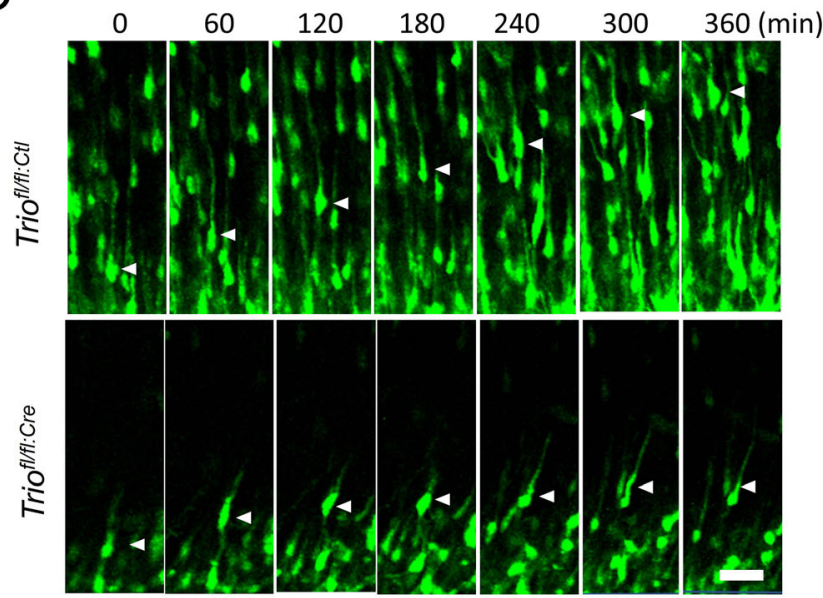

Fig. 2 Trio ablation leads to abnormal morphological transition and migratory behavior of cortical projection neurons. A Left panels, representative images of $\mathrm{GFP}^{+}$neuronal morphology (white asterisks) at E16.5 in Trio $^{\text {fl/f }}$ brains electroporated with either pcAGGS-CreIRES-GFP or a control vector at E14.5 (dotted boxes over the IZ are magnified on right) Scale bars, $50 \mu \mathrm{m}$. Right panel, percentages of unipolar/bipolar and multipolar neurons in each condition $(n=16$ slices from 3 Trio $^{\text {flffl:Ctl }}$ mice and $n=16$ slices from 3 Trio ${ }^{\text {flff:Cre }}$ mice).. B Representative images and quantification of the proportion of neurons with GRASP65 facing the CP in the IZ 2 days after E14.5

Trio-mediated radial migration, especially the decreased migration rate in Trio $^{f l f f: C r e}$ BP-shaped neurons. Among many Trio interaction candidates, we focused on the membrane delivery protein Myosin X, as it is involved in radial migration and signal transduction. TRIO9S coimmunoprecipitated with MYOSIN X in HEK293T cells (Fig. 4A). TRIO9S was constructed with seven spectrin repeat domain (SP) deletion (TRIO9S $\triangle \mathrm{SP}$ ), GEF1 and SH3 domain deletion (TRIO9S $\triangle$ GEF1-SH3), GEF1 domain deletion (TRIO9S $\triangle \mathrm{GEF} 1$ ), or GEF2 domain deletion
B

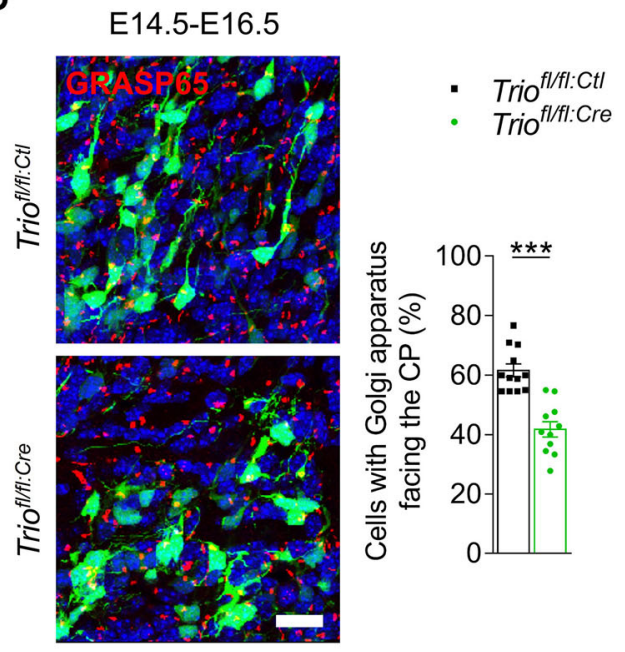

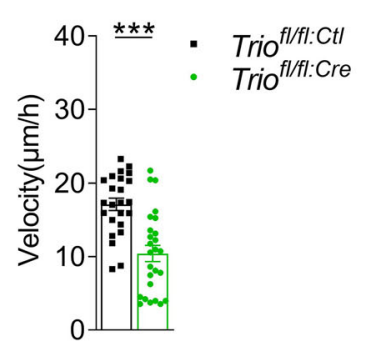

electroporation in Trio ${ }^{f l f t: C t l}$ and Trio ${ }^{f l f l: C r e}$ cells $(n=12$ slices from 3 Trio $^{f l f l: C t l}$ mice and $n=11$ slices from 3 Trio $^{f l f t: C r e}$ mice). Scale bar, $20 \mu \mathrm{m}$. C Left panels, representative images of time-lapse recording of migrating neurons in cultured cortical tissue after electroporation with the indicated plasmids. Arrowheads, migrating $\mathrm{EGFP}^{+}$neurons. Scale bar, $20 \mu \mathrm{m}$. Right panel, speed of migrating $\mathrm{EGFP}^{+}$neurons in each condition ( $n=24$ Trio $^{\text {flft:Ctl }}$ neurons and $n=26$ Trio $^{\text {flft:Cre }}$ neurons). Histograms show the mean \pm SEM. $* P<0.05$, $* * P<0.01$ *** $P<0.001$, Student's $t$ test.

(TRIO9S $\triangle$ GEF2; Fig. 4B). The binding affinities of the numerous TRIO9S domain truncations were used to determine the region of TRIO9S necessary for interaction with MYOSIN X. We found that the SH3 domain, but not the GEF1, GEF2, or SP domain, was important for the interaction of TRIO9S with MYOSIN X (Fig. 4C). We coelectroporated MYOSIN X and Cre into the developing Trio $^{f / f l}$ cortex to determine whether Myosin X overexpression rescued the migration defect caused by the loss of Trio. Myosin X overexpression partly rescued the 


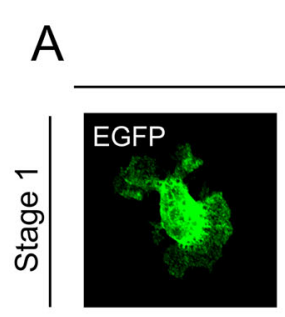

Trio flffl:ctl

Trio flffl:cre
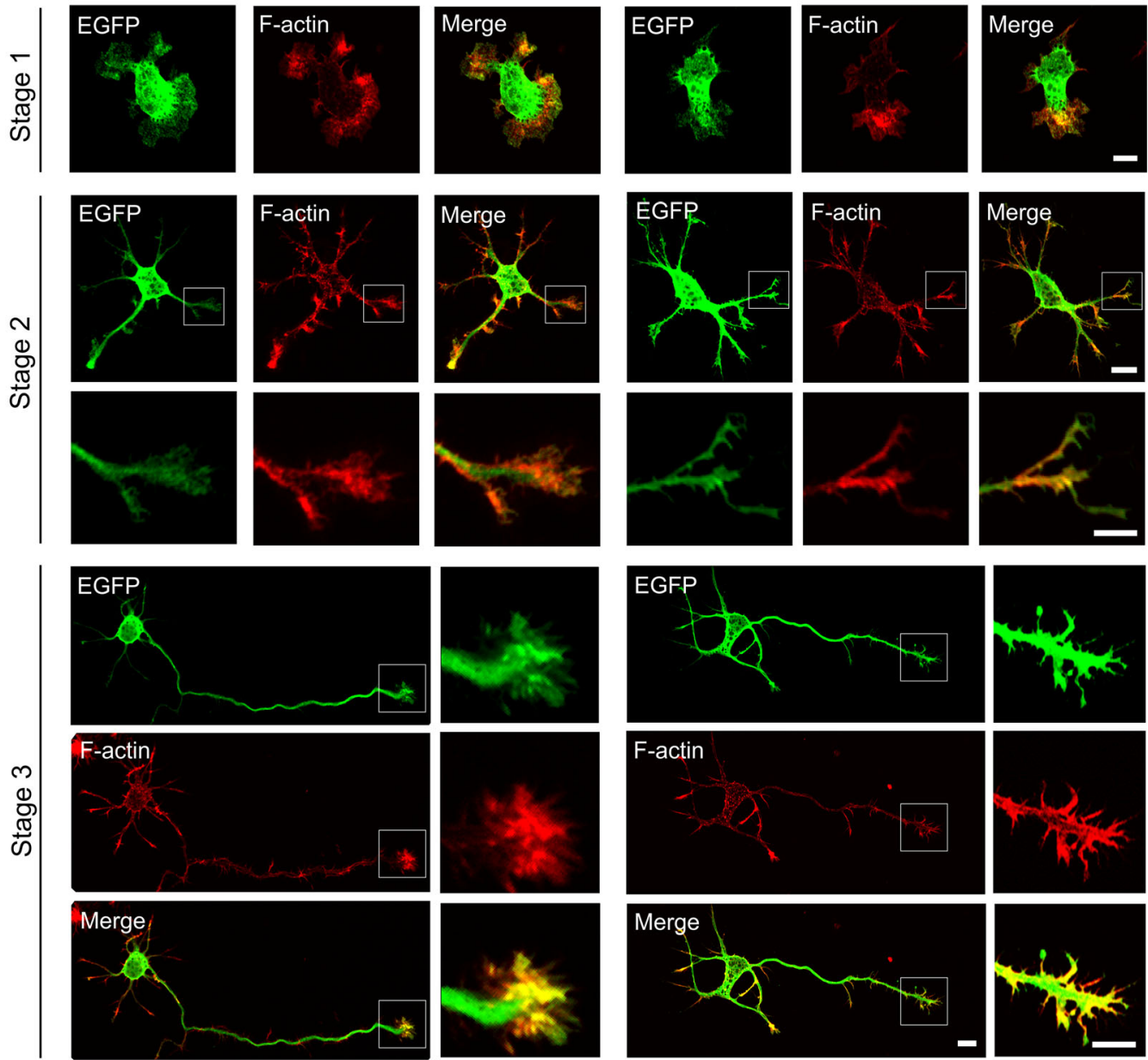

B

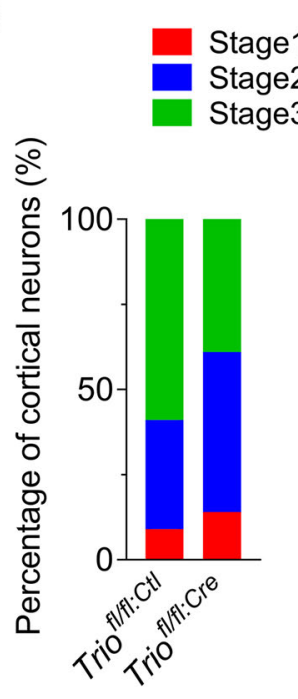

C
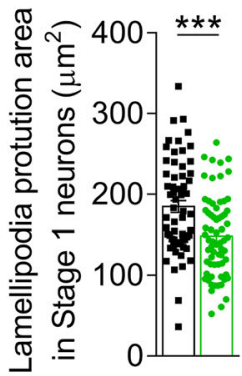

D

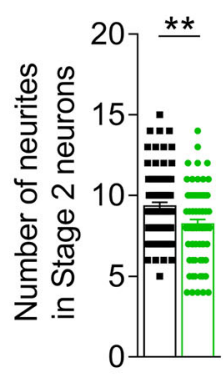

E

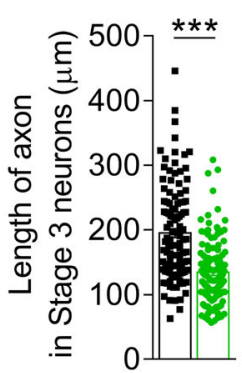

F

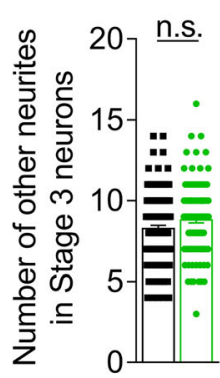

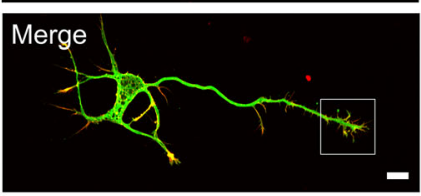

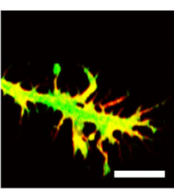


4 Fig. 3 Trio ablation leads to decreased polymerization of F-actin and abnormal neuronal polarization. A Representative images showing polymerization of F-actin in electroporated Trio ${ }^{f l f l: C r e}$ and Trio $o^{f / f l: C t l}$ neurons in vitro at stages 1,2 , and 3 . Scale bars, $10 \mu \mathrm{m}$ (stage 1 , stage 2 upper panel, and stage 3 left panel) and $5 \mu \mathrm{m}$ (stage 2 lower panel and stage 3 right panel). B Percentages of Trio ${ }^{f l f t: C r e}$ and Trio ${ }^{f l f t: C t l}$ neurons at different stages. C Area of lamellipodia protrusion in stage 1 neurons in the two groups. D Number of neurites in stage 2 neurons in the two groups. $\mathbf{E}, \mathbf{F}$ Length of axons $(\mathbf{E})$ and the number of other neurites $(\mathbf{F})$ in stage 3 Trio $^{f l f l: C r e}$ and Trio ${ }^{f / f l: C t l}$ neurons. Histograms show the mean \pm SEM. $* P<0.05$, $* * P<0.01$, $* * * P<0.001$; n.s., no significant difference, Student's $t$ test.

migration defects in Trio-deleted neurons (Fig. 4D). During radial migration to the $\mathrm{CP}$, the disruption of the radial glial fibers or the attachment of migrating neurons to the fiber led to abnormal neuronal migration. Nestin immunofluorescence revealed no abnormal morphology of radial glial fibers in Trio $^{f l f t: C r e}$ slices (Fig. S3A, B). The attachment of electroporated BP-shaped neurons to Nestin-positive $\left(\mathrm{Nestin}^{+}\right)$radial glial fibers in the upper IZ and lower CP regions was analyzed (Fig. 4E). The vertical distance between the center of the soma of $\mathrm{EGFP}^{+} \mathrm{BP}$ neurons and the Nestin ${ }^{+}$radial glial fibers was measured to assess the characteristics of the neuronal adhesion. Trio deficiency led to an increase in the distance between the center of the soma of BP neurons and the radial glial fibers compared with that measured in control cells. The overexpression of MYOSIN X rescued the defect, and the distance was similar to that measured in controls (Fig. 4F). Myosin X regulates the subcellular distribution of $\mathrm{N}$-cadherin, promoting $\mathrm{N}$-cadherin trafficking from the Golgi apparatus and endosomal sorting of vesicles to the plasma membrane [33]. Immunofluorescent staining revealed $\mathrm{N}$-cadherin expression on the plasma membrane of E17.5 neurons in each group. The N-cadherin levels on the plasma membrane located between two adjacent $\mathrm{EGFP}^{+}$neurons were quantified and normalized to that on the plasma membrane between adjacent EGFP-negative neurons. Strikingly, the expression of $\mathrm{N}$-cadherin at the cell borders between two adjacent $\mathrm{EGFP}^{+}$neurons was reduced in Trio-ablated neurons compared with the control group. The overexpression of Myosin $\mathrm{X}$ rescued the decreased membrane expression of N-cadherin (Fig. 4G). We also found the decreased density of $\mathrm{N}$-cadherin at the contact sites of migrating neurons and radial fibers in the Trio ${ }^{f l f t: C r e}$ neurons, and Myosin X overexpression rescued the abnormal distribution of $\mathrm{N}$-cadherin in the Trio ${ }^{f l f l: C r e}$ neurons (Fig. 4H). These data suggested that the Trio-Myosin X complexes promote neural adhesion and migration along radial glial fibers through $\mathrm{N}$-cadherin expression at the cell surface of cortical projection neurons.

\section{Overexpression of RAC1 and RHOA Rescues the Radial Migration Impairment by the Loss of Trio}

As shown in Fig. 4D, Myosin X overexpression in Triodeleted neurons had a limited effect during radial migration, suggesting that Trio regulates radial migration through other collaborative pathways. The levels of active Rac1, Cdc42, and RhoA, as effectors of Trio, were significantly reduced in the Trio-deficient brain at P0.5 [14]. Rac1 and RhoA were overexpressed in Trio-deleted neurons to explore the effect of downstream GTPase on Trio-mediated radial migration (Fig. 5A). Similar to Myosin X overexpression, Rac1 overexpression promoted the migration of Trio-deleted neurons from the IZ to the CP. Moreover, the proportion of Trio ${ }^{\text {flfl:Cre }}$ neurons in the upper CP increased upon the expression of RAC1. RhoA overexpression increased the migration of Trio ${ }^{\text {flfl:Cre }}$ neurons from the IZ to the CP. Simultaneously, more neurons migrated from the lower $\mathrm{CP}$ to the upper $\mathrm{CP}$. In addition, co-overexpressed Rac1 and RhoA effectively rescued the migratory defects of Trio-ablated neurons (Fig. 5B). The CA forms of PAK1 and ROCK [34, 35], the Rac1 and RhoA corresponding effector kinases, were

Fig. 4 Trio promotes radial migration through Myosin X-dependent $\mathrm{N}$-cadherin neuronal adhesion. A Interaction between exogenous TRIO9S and MYOSIN in lysates of HEK293T cells transfected with the indicated plasmids and immunoprecipitated. B Schematic of the TRIO9S domains and truncated mutants. C Immunoblots of coimmunoprecipitants by the full-length and numerous TRIO9S domain deletions with Myosin X. D Left panels, representative images of E17.5 embryonic cerebral cortex electroporated with indicated plasmids at E14.5. Scale bar, $50 \mu \mathrm{m}$. Right panel, distribution of $\mathrm{EGFP}^{+}$cells in the VZ/SVZ, IZ, lower CP, and upper CP in each condition. Histograms show the mean \pm SEM from at least 12 slices from 3 brains. E Representative images of $\mathrm{EGFP}^{+}$cells electroporated with indicated plasmids and $\mathrm{Nestin}^{+}$radial glial fibers (red). Scale bar, $5 \mu \mathrm{m}$. F Schematic of quantitative analysis (left panel), and the average distance between the center of the soma of the leading process and the $\mathrm{Nestin}^{+}$radial glial fiber in each condition (right panel). Histograms show the mean \pm SEM from at least 30 cells from 3 mice for each group. G Left panel, E17.5 cortical sections electroporated at E14.5 with indicated plasmids immunostained with $\mathrm{N}$-cadherin antibody. Scale bar, $5 \mu \mathrm{m}$. Middle panels, representative expression profiles of $\mathrm{N}$-cadherin measured across the cell bodies of paired $\mathrm{EGFP}^{+}$neurons. Right panel, the relative adjacent $\mathrm{N}$-cadherin signal quantified at the first $\mathrm{EGFP}^{-} / \mathrm{EGFP}^{+}$cell membrane/border (yellow arrowheads, as negative-EGFP), intermediate $\mathrm{EGFP}^{+} / \mathrm{EGFP}^{+}$ cell membrane/border (purple arrowheads, as EGFP-EGFP), and last $\mathrm{EGFP}^{+} / \mathrm{EGFP}^{-}$cell membrane/border (yellow arrowheads, as EGFPnegative) of two adjacent $\mathrm{EGFP}^{+}$cells for each condition. The first $\mathrm{EGFP}^{-} / \mathrm{EGFP}^{+}$cell membrane/border adjacent $\mathrm{N}$-cadherin signal was used for normalization. $\mathbf{H ~ N}$-cadherin signal density at the contact sites of migrating $\mathrm{EGFP}^{+}$neurons against radial fibers under every condition. Histograms show the mean \pm SEM from at least 20 neurons from 3 brains for each group. $* P<0.05$, $* * P<0.01$, $* * * P<0.001$, n.s., no significant difference, Student's $t$ test. 
A

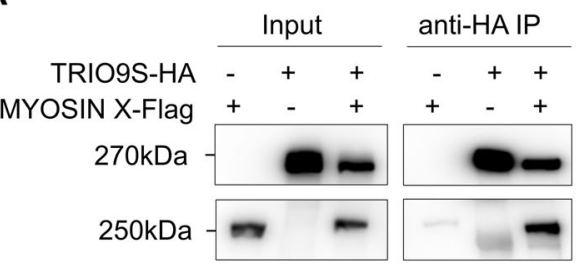

C

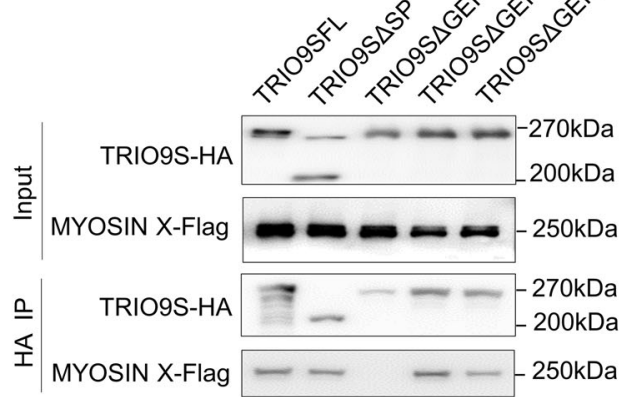

E
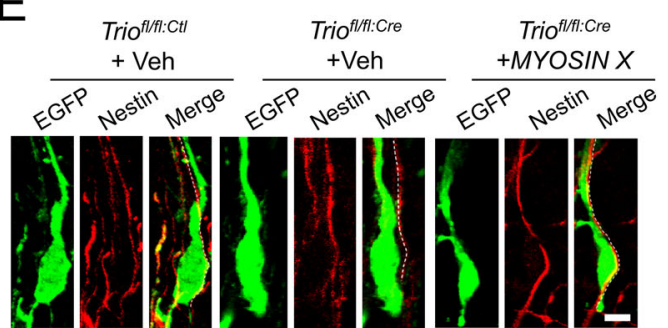

G

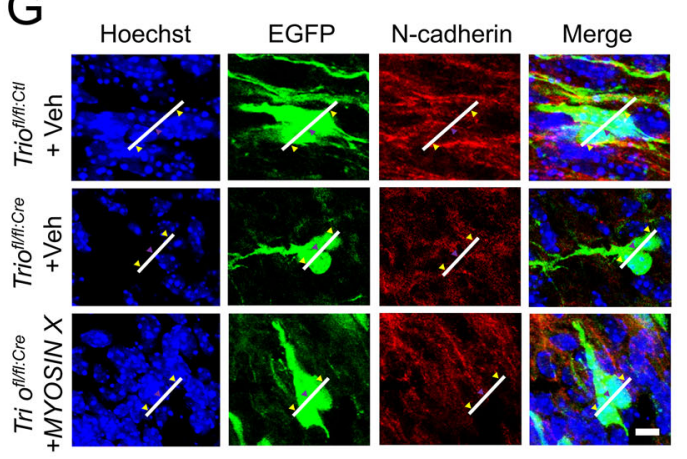

B

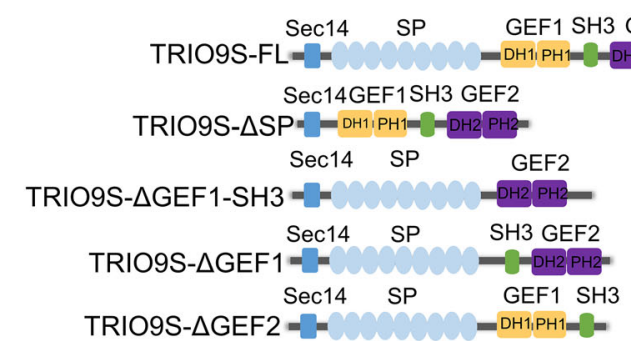

D
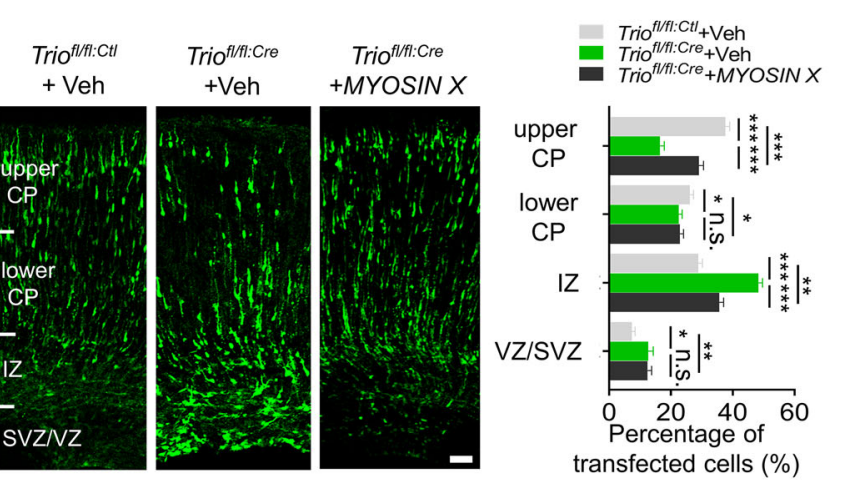

F
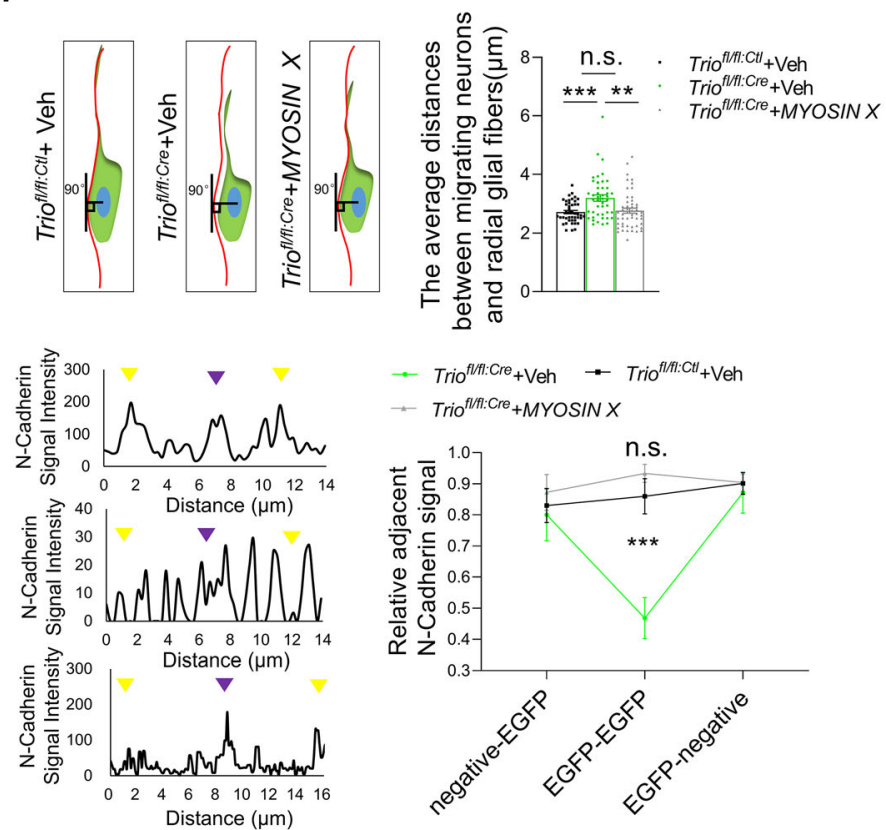

$\mathrm{H}$

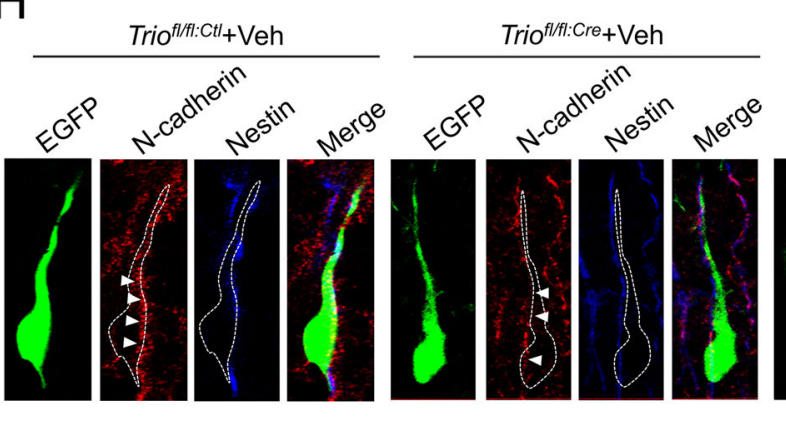

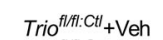

Trio flff:Cre + Veh Trio ${ }^{\text {flff:Cre }}+$ MYOSINX

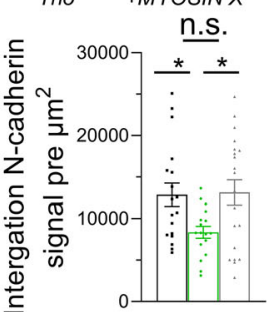



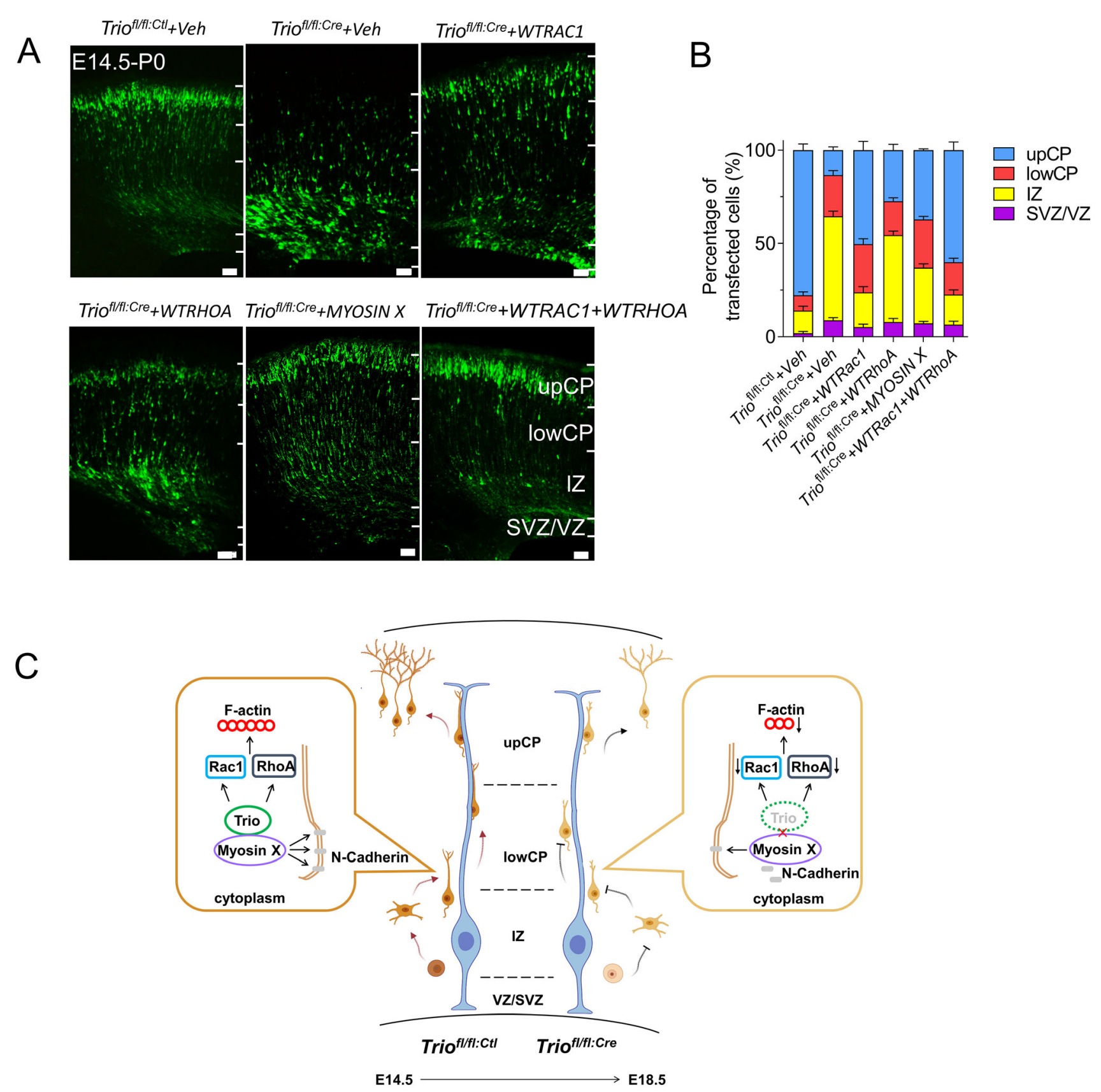

Fig. 5 Defects of neuronal migration are rescued by overexpressed RAC1 and ROHA in Trio-ablated cells. A Co-electroporation of pcAGGS-Cre-IRES-EGFP with RAC1, RHOA, MYOSIN X, RAC1, and RhoA into E14.5 Trio $^{f l f l}$ brains at E18.5; the pcAGGS-IRESEGFP plasmid serves as control. Scale bars, $50 \mu \mathrm{m}$. B Relative distributions (percentages) of EGFP-expressing cells in the VZ/SVZ,

electroporated into Trio-deleted neurons to investigate further the effects of decreased Rho GTPase activity caused by Trio deletion. Although CA-PAK1 overexpression in Trio-deleted neurons failed to rescue the migratory defects, CA-ROCK overexpression promoted more Trio-
$\mathrm{IZ}$, lower $\mathrm{CP}$, and upper $\mathrm{CP}$ under each condition as in (A). Histogram shows the mean \pm SEM from at least 12 slices from 3 brains. C Model of the role of Trio in the migration of cortical projection neurons. Trio ablation affects the multipolar-bipolar transition by regulating F-actin dynamics and the radial glial fiber relative migration by locating cell membrane $\mathrm{N}$-cadherin in late-born neurons.

deleted neurons from stagnation in the IZ into the $\mathrm{CP}$ (Fig. S4). These results suggested that the reduced activation of Rac1 inhibits both the MP-to-BP transition and radial glial-dependent migration, while the reduced 
activation of RhoA restricts the migration of cells located in the lower $\mathrm{CP}$ into the upper $\mathrm{CP}$ in Trio-deficient neurons.

\section{Discussion}

In this study, we investigated the role of Trio in cortical radial migration. Our results revealed that Trio was essentially involved in neuronal morphogenesis and the MP-to-BP transition of late-born PNs. Furthermore, Trio interacted with Myosin X through its $\mathrm{N}$-terminal SH3 domain, which regulated the membrane location of $\mathrm{N}$-cadherin and was critical for the neuronal adhesion and migration along radial glial fibers. As Trio is a Rho GEF, the activation of Rac1 and RhoA mediated by the GEF1 and GEF2 domains played independent and synergistic roles in the radial migration of PNs. These findings suggested the vital role of Trio-related signaling in the cortical radial migration of PNs.

The homozygous Trio knockout in mice is lethal during embryonic development, as previously reported [36]. Thus, in utero electroporation and Emxl-mediated conditional Trio deletion were used to study the radial migration during embryonic development. Our results suggested that Trio deletion affected both the MP-to-BP transition and glialguided migration in late-born PNs but not the migration of early-born neurons. In addition, Trio ablation in early postmitotic PNs resulted in neuronal migration deficits similar to Trio ablation in VZ neural progenitors, indicating the importance of Trio in the migration of postmitotic neurons. However, the role of Trio in neural progenitors during development cannot be ignored and deserves further study, although the deletion of Trio has no effect on proliferation or apoptosis.

Similar to the Trio-ablation-induced radial migration deficits reported in this study, previous studies have demonstrated that Rac1 knockdown or inhibition of its activity with N17-Rac1 blocks radial migration and disrupts the formation of the leading process [37-39]. Meanwhile, the overexpression of wild-type RAC1 facilitated the migration of Trio-deleted neurons from the IZ to the lower CP, further highlighting the vital role of Rac1 in radial migration. However, in accordance with the effect of CA-Rac1 overexpression on neuronal migration [38, 39], the overexpression of a constitutively active form of PAK1, the effector kinase of active Rac1 for regulating F-actin dynamics [40-43], showed a poorer migratory behavior in Trio-deleted neurons, indicating that the appropriate activity of Rac1-Pak1 signaling is vital for neuronal polarity in radial migration. However, the overexpression of wild-type RhoA or a constitutively active form of ROCK in Trioablated neurons in the present study facilitated the migration of more IZ-trapped cells to the $\mathrm{CP}$, which is inconsistent with previous findings [44-47]. This might be attributed to the different genetic context of Trio deletion in this study, in which the activities of Racl and RhoA were affected simultaneously. Comprehensively, our findings provided evidence that Trio facilitates Rac1 and RhoA signaling to maintain the morphological transition, migration, and adhesion of cortical projection neurons. Trio GEF1 and GEF2 might have distinct and cooperative actions on their Rho GTPase effectors to promote the radial migration of PNs.

Endocytosis and recycling of $\mathrm{N}$-cadherin in migratory neurons promote the interaction between neurons and radial glial fibers in neuronal radial locomotion [48]. The suppression of Myosin X via in utero electroporation led to the migration of a reduced number of neurons in the $\mathrm{CP}$ and the accumulation of neurons in the IZ due to abnormal $\mathrm{N}$-cadherin distribution into the Golgi apparatus and endosomal sorting vesicles [33]. Previous studies demonstrated that both Myosin X and Trio interact with DCC (deleted in colorectal cancer), a receptor of Netrin-1 [10, 49], and the Rac1 activation elicited by Netrin- 1 is lost in the Trio-deficient cortex [10]. Otherwise, RhoA and Rock promote Myosin activation by phosphorylating the Myosin light chain to regulate cell polarity, cell migration, and cell-cell adhesion junctions in some cell types [50-54]. However, the relationship between Myosin X and Triomeditated signaling is largely unknown. In this study, we found that Trio was associated with Myosin X. The overexpression of Myosin X facilitated the migration of Trio-deleted neurons into the upper $\mathrm{CP}$ and a tight attachment to radial glial fibers through regulating the membrane localization of $\mathrm{N}$-cadherin, indicating that Triomediated Rac1 and RhoA signaling is involved in the Myosin X-mediated membrane location of $\mathrm{N}$-cadherin in radial migration.

Taken together, our results provide evidence for a novel mechanism of $\mathrm{N}$-cadherin cell surface expression regulated by the Trio-Myosin X complex. The GEF1 and GEF2 domains of Trio and the related signaling have distinct and cooperative actions in regulating radial migration by activating their Rho GTPase effectors.

Acknowledgements This work was supported by grants from the Key Realm R\&D Program of Guangdong Province (2019B030335001), the National Key R\&D Program of China (2016YFC1307000), the National Natural Science Foundation of China (81730037, 81825009, 81871077, 81671363, and 82071541), and the Beijing Major Science and Technology Projects (Z181100001518001).

Conflict of interest The authors declare no conflict of interest.

Open Access This article is licensed under a Creative Commons Attribution 4.0 International License, which permits use, sharing, adaptation, distribution and reproduction in any medium or format, as 
long as you give appropriate credit to the original author(s) and the source, provide a link to the Creative Commons licence, and indicate if changes were made. The images or other third party material in this article are included in the article's Creative Commons licence, unless indicated otherwise in a credit line to the material. If material is not included in the article's Creative Commons licence and your intended use is not permitted by statutory regulation or exceeds the permitted use, you will need to obtain permission directly from the copyright holder. To view a copy of this licence, visit http://creativecommons. org/licenses/by/4.0/.

\section{References}

1. Silva CG, Peyre E, Nguyen L. Cell migration promotes dynamic cellular interactions to control cerebral cortex morphogenesis. Nat Rev Neurosci 2019, 20: 318-329.

2. LoTurco JJ, Bai J. The multipolar stage and disruptions in neuronal migration. Trends Neurosci 2006, 29: 407-413.

3. Nadarajah B, Brunstrom JE, Grutzendler J, Wong RO, Pearlman AL. Two modes of radial migration in early development of the cerebral cortex. Nat Neurosci 2001, 4: 143-150.

4. Yang DY, Beam D, Pelphrey KA, Abdullahi S, Jou RJ. Cortical morphological markers in children with autism: A structural magnetic resonance imaging study of thickness, area, volume, and gyrification. Mol Autism 2016, 7: 11.

5. Muraki K, Tanigaki K. Neuronal migration abnormalities and its possible implications for schizophrenia. Front Neurosci 2015, 9: 74.

6. Peñagarikano $\mathrm{O}$, Abrahams BS, Herman EI, Winden $\mathrm{KD}$, Gdalyahu A, Dong HM. Absence of CNTNAP2 leads to epilepsy, neuronal migration abnormalities, and core autism-related deficits. Cell 2011, 147: 235-246.

7. Pan $\mathrm{YH}, \mathrm{Wu} \mathrm{N}$, Yuan XB. Toward a better understanding of neuronal migration deficits in autism spectrum disorders. Front Cell Dev Biol 2019, 7: 205.

8. Küry S, van Woerden GM, Besnard T, Proietti Onori M, Latypova X, Towne MC, et al. De novo mutations in protein kinase genes CAMK2A and CAMK2B cause intellectual disability. Am J Hum Genet 2017, 101: 768-788.

9. DeGeer J, Kaplan A, Mattar P, Morabito M, Stochaj U, Kennedy TE, et al. Hsc70 chaperone activity underlies Trio GEF function in axon growth and guidance induced by netrin-1. J Cell Biol 2015, 210: 817-832.

10. Briançon-Marjollet A, Ghogha A, Nawabi H, Triki I, Auziol C, Fromont $\mathrm{S}$, et al. Trio mediates netrin-1-induced Rac1 activation in axon outgrowth and guidance. Mol Cell Biol 2008, 28: 2314-2323.

11. Ba W, Yan Y, Reijnders MR, Schuurs-Hoeijmakers JH, Feenstra I, Bongers EM, et al. TRIO loss of function is associated with mild intellectual disability and affects dendritic branching and synapse function. Hum Mol Genet 2016, 25: 892-902.

12. Katrancha SM, Shaw JE, Zhao AY, Myers SA, Cocco AR, Jeng AT, et al. Trio haploinsufficiency causes neurodevelopmental disease-associated deficits. Cell Rep 2019, 26: 2805-2817.e9.

13. Zong W, Liu SY, Wang XT, Zhang J, Zhang TT, Liu ZY, et al. Trio gene is required for mouse learning ability. Brain Res 2015, 1608: 82-90.

14. Peng YJ, He WQ, Tang J, Tao T, Chen C, Gao YQ, et al. Trio is a key guanine nucleotide exchange factor coordinating regulation of the migration and morphogenesis of granule cells in the developing cerebellum. J Biol Chem 2010, 285: 24834-24844.

15. Guo DJ, Yang XM, Shi L. Rho GTPase regulators and effectors in autism spectrum disorders: Animal models and insights for therapeutics. Cells 2020, 9: E835.
16. Sadybekov A, Tian C, Arnesano C, Katritch V, Herring BE. An autism spectrum disorder-related de novo mutation hotspot discovered in the GEF1 domain of Trio. Nat Commun 2017, 8: 601.

17. Barbosa S, Greville-Heygate S, Bonnet M, Godwin A, FagottoKaufmann C, Kajava AV, et al. Opposite modulation of RAC1 by mutations in TRIO is associated with distinct, domain-specific neurodevelopmental disorders. Am J Hum Genet 2020, 106: 338-355.

18. Schultz-Rogers L, Muthusamy K, Pinto E, Vairo F, Klee EW, Lanpher B. Novel loss-of-function variants in TRIO are associated with neurodevelopmental disorder: Case report. BMC Med Genet 2020, 21: 219.

19. Blangy A, Vignal E, Schmidt S, Debant A, Gauthier-Rouvière C, Fort P. TrioGEF1 controls Rac- and Cdc42-dependent cell structures through the direct activation of rhoG. J Cell Sci 2000, 113(Pt 4): 729-739.

20. Bellanger JM, Lazaro JB, Diriong S, Fernandez A, Lamb N, Debant A. The two guanine nucleotide exchange factor domains of Trio link the Rac1 and the RhoA pathways in vivo. Oncogene 1998, 16: 147-152.

21. Chhatriwala MK, Betts L, Worthylake DK, Sondek J. The DH and PH domains of Trio coordinately engage Rho GTPases for their efficient activation. J Mol Biol 2007, 368: 1307-1320.

22. Bellanger JM, Astier C, Sardet C, Ohta Y, Stossel TP, Debant A. The Rac1- and RhoG-specific GEF domain of Trio targets filamin to remodel cytoskeletal actin. Nat Cell Biol 2000, 2: 888-892.

23. van Rijssel J, Hoogenboezem M, Wester L, Hordijk PL, van Buul JD. The N-terminal DH-PH domain of Trio induces cell spreading and migration by regulating lamellipodia dynamics in a Rac1-dependent fashion. PLoS ONE 2012, 7: e29912. https:// doi.org/10.1371/journal.pone.0029912.

24. Schiller MR, Chakrabarti K, King GF, Schiller NI, Eipper BA, Maciejewski MW. Regulation of RhoGEF activity by intramolecular and intermolecular SH3 domain interactions. J Biol Chem 2006, 281: 18774-18786.

25. Tabata H, Nakajima K. Efficient in utero gene transfer system to the developing mouse brain using electroporation: Visualization of neuronal migration in the developing cortex. Neuroscience 2001, 103: 865-872.

26. Guerrier S, Coutinho-Budd J, Sassa T, Gresset A, Jordan NV, Chen K, et al. The F-BAR domain of srGAP2 induces membrane protrusions required for neuronal migration and morphogenesis. Cell 2009, 138: 990-1004.

27. de Anda FC, Pollarolo G, da Silva JS, Camoletto PG, Feiguin F, Dotti CG. Centrosome localization determines neuronal polarity. Nature 2005, 436: 704-708.

28. Jacobson C, Schnapp B, Banker GA. A change in the selective translocation of the Kinesin-1 motor domain marks the initial specification of the axon. Neuron 2006, 49: 797-804.

29. McPherson CE, Eipper BA, Mains RE. Multiple novel isoforms of Trio are expressed in the developing rat brain. Gene 2005, 347: $125-135$.

30. Ma XM, Huang JP, Eipper BA, Mains RE. Expression of Trio, a member of the Dbl family of Rho GEFs in the developing rat brain. J Comp Neurol 2005, 482: 333-348.

31. Yoshizuka N, Moriuchi R, Mori T, Yamada K, Hasegawa S, Maeda $\mathrm{T}$, et al. An alternative transcript derived from the trio locus encodes a guanosine nucleotide exchange factor with mouse cell-transforming potential. J Biol Chem 2004, 279: 43998-44004.

32. Katrancha SM, Wu Y, Zhu MS, Eipper BA, Koleske AJ, Mains RE. Neurodevelopmental disease-associated de novo mutations and rare sequence variants affect TRIO GDP/GTP exchange factor activity. Hum Mol Genet 2017, 26: 4728-4740. 
33. Lai MM, Guo Y, Ma J, Yu HL, Zhao DD, Fan WQ, et al. Myosin $\mathrm{X}$ regulates neuronal radial migration through interacting with N-cadherin. Front Cell Neurosci 2015, 9: 326.

34. Sells MA, Boyd JT, Chernoff J. p21-activated kinase 1 (Pak1) regulates cell motility in mammalian fibroblasts. J Cell Biol 1999, 145: 837-849.

35. Chang J, Xie M, Shah VR, Schneider MD, Entman ML, Wei L, et al. Activation of Rho-associated coiled-coil protein kinase 1 (ROCK-1) by caspase-3 cleavage plays an essential role in cardiac myocyte apoptosis. Proc Natl Acad Sci U S A 2006, 103 : 14495-14500.

36. O’Brien SP, Seipel K, Medley QG, Bronson R, Segal R, Streuli M. Skeletal muscle deformity and neuronal disorder in Trio exchange factor-deficient mouse embryos. Proc Natl Acad Sci U S A 2000, 97: 12074-12078.

37. Kawauchi T, Chihama K, Nabeshima Y, Hoshino M. The in vivo roles of STEF/Tiam1, Rac1 and JNK in cortical neuronal migration. EMBO J 2003, 22: 4190-4201.

38. Konno D, Yoshimura S, Hori K, Maruoka H, Sobue K. Involvement of the phosphatidylinositol 3-kinase/rac1 and cdc42 pathways in radial migration of cortical neurons. J Biol Chem 2005, 280: 5082-5088.

39. Yang T, Sun YM, Zhang F, Zhu YG, Shi L, Li HS, et al. POSH localizes activated Rac1 to control the formation of cytoplasmic dilation of the leading process and neuronal migration. Cell Rep 2012, 2: 640-651.

40. Meyer Zum Büschenfelde U, Brandenstein LI, von Elsner L, Flato K, Holling T, Zenker M, et al. RIT1 controls actin dynamics via complex formation with RAC1/CDC42 and PAK1. PLoS Genet 2018, 14: e1007370. https://doi.org/10.1371/journal. pgen.1007370.

41. Vadlamudi RK, Li F, Barnes CJ, Bagheri-Yarmand R, Kumar R. p41-Arc subunit of human Arp2/3 complex is a p21-activated kinase-1-interacting substrate. EMBO Rep 2004, 5: 154-160.

42. Kubo Y, Baba K, Toriyama M, Minegishi T, Sugiura T, Kozawa $\mathrm{S}$, et al. Shootin1-cortactin interaction mediates signal-force transduction for axon outgrowth. J Cell Biol 2015, 210: 663-676.

43. Staser K, Shew MA, Michels EG, Mwanthi MM, Yang FC, de Clapp DW, et al. A Pak1-PP2A-ERM signaling axis mediates F-actin rearrangement and degranulation in mast cells. Exp Hematol 2013, 41: 56-66.e2.

44. Tang J, Ip JPK, Ye T, Ng YP, Yung WH, Wu Z, et al. Cdk5dependent Mst3 phosphorylation and activity regulate neuronal migration through RhoA inhibition. J Neurosci 2014, 34: 7425-7436.

45. Nguyen L, Besson A, Heng JI, Schuurmans C, Teboul L, Parras C, et al. p27Kip1 independently promotes neuronal differentiation and migration in the cerebral cortex. Bull Mem Acad R Med Belg 2007, 162: 310-314.

46. Pacary E, Heng J, Azzarelli R, Riou P, Castro D, Lebel-Potter M, et al. Proneural transcription factors regulate different steps of cortical neuron migration through Rnd-mediated inhibition of RhoA signaling. Neuron 2011, 69: 1069-1084.

47. Azzarelli R, Pacary E, Garg R, Garcez P, van den Berg D, Riou P, et al. An antagonistic interaction between PlexinB2 and Rnd3 controls RhoA activity and cortical neuron migration. Nat Commun 2014, 5: 3405.

48. Shikanai M, Nakajima K, Kawauchi T. N-cadherin regulates radial glial fiber-dependent migration of cortical locomoting neurons. Commun Integr Biol 2011, 4: 326-330.

49. Zhu XJ, Wang CZ, Dai PG, Xie Y, Song NN, Liu Y, et al. Myosin X regulates netrin receptors and functions in axonal pathfinding. Nat Cell Biol 2007, 9: 184-192.

50. Kimura K, Ito M, Amano M, Chihara K, Fukata Y, Nakafuku M, et al. Regulation of myosin phosphatase by Rho and Rhoassociated kinase (Rho-kinase). Science 1996, 273: 245-248.

51. Gutjahr MC, Rossy J, Niggli V. Role of Rho, Rac, and Rhokinase in phosphorylation of myosin light chain, development of polarity, and spontaneous migration of Walker 256 carcinosarcoma cells. Exp Cell Res 2005, 308: 422-438.

52. Totsukawa G, Wu Y, Sasaki Y, Hartshorne DJ, Yamakita Y, Yamashiro S, et al. Distinct roles of MLCK and ROCK in the regulation of membrane protrusions and focal adhesion dynamics during cell migration of fibroblasts. J Cell Biol 2004, 164: 427-439.

53. Priya R, Gomez GA, Budnar S, Verma S, Cox HL, Hamilton NA, et al. Feedback regulation through myosin II confers robustness on RhoA signalling at E-cadherin junctions. Nat Cell Biol 2015, 17: 1282-1293.

54. Quiros M, Nusrat A. RhoGTPases, actomyosin signaling and regulation of the epithelial Apical Junctional Complex. Semin Cell Dev Biol 2014, 36: 194-203. 